\title{
Testicular Fine Needle Aspiration in Evaluation of Male Infertility
}

\author{
Jha ${ }^{\prime},{ }^{\prime}$ Sayami $\mathrm{G}^{1}$ \\ 'Department of Pathology, Tribhuvan University Teaching Hospital, Kathmandu, Nepal
}

\begin{abstract}
Fine needle aspiration cytology of superficial as well as of deep seated lesions is now well recognized in diagnosis of neoplastic as well as nonneoplastic and inflammatory lesions. Recently it has also gained popularity for its diagnostic and therapeutic role in male infertility. The purpose of this article was to review various studies published on role of testicular fine needle testicular cytology in male infertility and provide a brief information on method of testicular fine needle aspiration, interpretation of testicular fine needle aspiration cytology for evaluation of spermatogenesis, its advantages, limitations and complications as compared to testicular biopsy.
\end{abstract}

Key words: cytology, fine needle aspiration, infertility, reproduction, spermatogenesis

\section{INTRODUCTION}

Approximately $20 \%$ cases of infertility are caused entirely by male factor with an additional $30 \%$ to $40 \%$ cases involving both male and female factors. ${ }^{1}$ Thus male factor is present in approximately $30 \%$ to $50 \%$ infertile couples. ${ }^{1-3}$ Azoospermia or absent sperm in semen occurs in approximately five to ten percent of infertile men who are evaluated. ${ }^{4}$ Azoospermia may be obstructive azoospermia or non obstructive azoospermia (NOA). The obstructive cause may have no significant effect on spermatogenesis and may be amenable to surgery where as before introduction of intracytoplasmic sperm injection (ICSI), the only available option for men with NOA was adoption or sperm donor.

Assessment of spermatogenesis is an important component in the diagnostic algorithm of male infertility. Traditionally, the surgical testis biopsy has been the gold standard in this evaluation because it provides information in cases of both suspected obstruction and in failing unobstructed testes. Any technique to assess spermatogenesis must be minimally invasive and must conserve as much testis tissue as possible. It should also not only provide qualitative but quantitative information about spermatogenesis. In addition to answering the question of whether sperm production is normal, it must also address whether any sperm are present at all within the testis, as with advances in field of reproductive medicine, even a single sperm can now give men with NOA chance to enjoy biological fatherhood. ${ }^{5}$

Testicular biopsy is well established as one of the main investigative modalities in male infertility for evaluation of spermatogenesis. But the tissue sample is small and not representative of entire testis. ${ }^{6}$ It is also invasive and traumatic especially when applied to both testes. ${ }^{7}$ Fine needle aspiration cytology (FNAC) of testes has proved to be useful in testicular tumors as well as in non neoplastic and inflammatory conditions of testes. ${ }^{6-9}$ However its greatest value is in evaluation

\footnotetext{
Correspondence:

Dr. Runa Jha

Department of Pathology,

Tribhuvan University Teaching Hospital

Maharajgung, Katmandu, Nepal

Email: runa75jha@yahoo.com

Phone: 9841368608
} 
Jha et al. Testicular Fine Needle Aspiration in Evaluation of Male Infertility

of spermatogenesis in azoospermic males, particularly in NOA, where it can conserve tissue of already failing organ.

Needle aspiration of testes was first described by Max Huhner, however it was later in 1965 only that first fine needle aspiration (FNA) of human testes in men with fertility disorder was performed by Scandinavian group pioneered by Obrant and Persson, still not fully describing the morphologic features at various stages of spermatogenesis. Later cytologic features of seminiferous epithelium was described by Schenk and Schill. ${ }^{2,7,10}$ However testicular FNA did not gain popularity then because of lack of experience of pathologists in cytologic analysis of various seminiferous tubular cells, because of fear of trauma to the testes with hematoma formation by the procedure and because cytology was unable to give information about the tubular basement membrane and status of interstitial tissue. ${ }^{11}$ But later on many studies carried out showed that FNAC evaluated spermatogenesis of entire testes, was less invasive, report could be issued quicker and there was good cytologic- histologic correlation. ${ }^{2,3,6,12-}$ 18 It was also concluded that information of tubular basement membrane and interstitial tissue was of no help in evaluation of spermatogenesis. ${ }^{7}$

Not only in diagnosis, but testicular FNA has also found therapeutic implication in assisted reproduction technique. Since the introduction of intracytoplasmic sperm injection (ICSI) in 1992, several studies of testicular sperm retrieval in azoospermic patients have been reported. ${ }^{19-21}$ These studies have encouraged the modifications in various sperm retrieval techniques. Because biopsy procedures remove interstitial, hormone-producing tissue non selectively with seminiferous tubules, substantial and possibly critical reductions in Leydig cell mass are possible and remain a concern in men who present with atrophic testes. ${ }^{22}$ Also, testicular open biopsies have side-effects like haematomas, inflammation and especially permanent devascularization of the testis resulting in testicular atrophy. ${ }^{17}$ So FNA of the testis in sperm retrieval have also started to gain popularity.

\section{FNA TECHNIQUE}

Testicular FNA is done under local anesthesia. ${ }^{6,7}$ The scrotal skin is cleaned and spermatic cord block is achieved by 5 to $7 \mathrm{ml}$ of two percent lidocaine. To quicken the distribution of anesthetic, spermatic cord is gently massaged after injection. After several minutes the testis is firmly palpated to ensure absence of pain. Then the testis is positioned with epididymis and vas deferens directed posteriorly, safe from injury. The scrotal skin is stretched taut over the testes by wrapping the scrotal skin behind the testes with a sponge. The testicular wrap serves not only as convenient handle to manipulate the testes but also fixes the scrotal skin over the testes for procedure. ${ }^{23}$ Testes is aspirated at three different sites, upper, middle and lower part, using 21-23 G needle with $10 \mathrm{ml}-20 \mathrm{ml}$ syringe attached to it. Precise gentle in and out movement, varying from $5-8 \mathrm{~mm}$ are used. Testes can also be needled without local anesthesia, but only at one site and procedure should be completed in 10-15 seconds. The patient should rest for at least ten minutes after the procedure. ${ }^{7}$ Both testes should be sampled when FNA is done for evaluation of spermatogenesis. Slides are prepared from the aspirated material and are fixed in alcohol and stained with Papanicolaou (Pap) stain or are air dried and stained with Geimsa stain. ${ }^{2,6,15}$ Staining the smears with Geimsa or Pap is not superior to each other. Both staining methods should be used together in order to use advantages of each method during the microscopic evaluation. ${ }^{17}$ Geimsa stain may be superior to Papanicolaou stain in defining cell borders of spermatogenic cells but tail of spermatozoa is most readily visible with Pap stain. ${ }^{24}$

\section{REPORTING TESTICULAR FNAC FOR EVALUATION OF SPERMITTOGENESIS}

\section{Specimen adequacy for FNA}

If at least 200 cells could be counted on minimum one well spread slide, specimen is considered adequate. ${ }^{2}$ Approximately $97 \%$ testicular FNA yield adequate specimen for evaluation of spermatogenesis. ${ }^{7} 200-500$ consecutive cells should be counted and percentage of different cells noted. Cytologic results are satisfactorily reproducible. ${ }^{11}$

Two cell populations are evident in cytology. ${ }^{6,15}$

- Sertoli cells

- Cells in various stages of spermatogenesis

The spermatogenetic cells are divided into spermatogonia, primary spermatocytes, secondary spermatocytes, spermatids and spermatozoa. Cytologic features of these cells are described below:

Sertoli cells: These cells have round vesicular nucleus with finely granular chromatin and large nucleolus. The nuclear outline is smooth. Cytoplasm is abundant, pale and vacuolated with poorly delineated border. These cells are fragile and so naked nuclei are common.

Spermatogonia: These are uninucleated mainly but may be binucleated or multinucleated. The nuclei are round or oval, slightly eccentric and dark or pale depending upon their chromatin density. The cytoplasm is homogenous and has well defined border. In air dried Geimsa stained smears the spermatogonia may resemble lymphomatoid blast. 
Jha et al. Testicular Fine Needle Aspiration in Evaluation of Male Infertility

Primary spermatocytes: These cells have large nucleus with thread like or coarse chromatin. Nuclear outline may be irregular. The cytoplasm if present is basophilic and it is more deeply stained at the periphery of the cell. Binucleated primary spermatocytes are common. Primary spermatocytes are either isolated or are present in groups with other spermatogenic cells or sertoli cells.

Secondary spermatocytes: These cells are rarely identified because of their shorter life span and immediate transformation to spermatids

Spermatids: They have much smaller nucleus than spermatocytes, corresponding to their haploid set of chromosomes. The nucleus is often centrally placed and finely granular. They may also be binucleated and have cytoplasmic vacuoles. They may be found in groups in normal testes, resembling sheets of epithelial cells.

Spermatozoa: They have oval nuclei with very dense chromatin. The tail is found on opposite side of acrosome.

Leydig cells: The aspiration of interstitial tissue is difficult and hence Leydig cells are usually not visualized in cytologic smears. If present, in smears they appear singly or in clusters. They are somewhat smaller than the sertoli cells and have central spherical or oval nucleus. Some are binucleated. They have abundant finely granular eosinophilic cytoplasm

\section{FNA interpretation}

Depending upon the percentage of different cells counted the result is interpreted as one of the following: ${ }^{3,7,16}$

- Normal spermatogenesis

- Hypospermatogenesis

- Sertoli cell only (SCO)

- Maturation arrest.

- Atrophic pattern

Normal spermatogenesis: Smears show abundant cellularity with 10-20 spermatozoa per 400x field (HPF). Abundant primary spermatocytes and spermatids are present. The ratio of spermatogenic to sertoli cell is at least 1.5:1 (60:40).

Hypospermatogenesis: Smears show relative decrease in all three germ cell types as compared to normal spermatogenesis. Less than 10 spermatozoa are visualized in each 400x field (HPF). Overall paucity of cell is seen, however all three kinds of germ cells are present including primary spermatocytes, spermatid and spermatozoa. Ratio of spermatogenic to sertoli cell is less than $1.5: 1$.
Sertoli cells only/Germ cell aplasia: Smears show mainly sertoli cells.

Maturation arrest: Maturation arrest is divided into early maturation arrest and late maturation arrest. In early (premeiotic) maturation arrest smears have adequate cellularity and show numerous primary spermatocytes. No or only occasional spermatids or spermatozoa are visualized. In Late maturation arrest (Post meiotic arrest) normal number of primary spermatocytes and spermatids are present but spermatozoa are not seen or are only occasionally seen.

Atrophic pattern: Smears show mainly proteinaceous material and scant sertoli and leydig cell.

Various cell indices can be calculated with the help of differential cell count. Useful indices are spermatic index (Spermatozoa/ all spermatogenetic cell), sertoli cell index (Sertoli cell / all spermatogenic cell) and sperm - sertoli cell index (spermatozoa/sertoli cells). Progressively increasing value of sertoli cell index and progressively decreasing value of sperm - sertoli cell index is detected in normal spermatogenesis, maturation arrest, hypospermatogenesis and sertoli only cell syndrome respectively. ${ }^{2,26}$

\section{CORRELATION BETWEEN FNAC AND HISTOLOGY IN EVALUATING SPERIMATOGENESIS}

Correlation between histology and cytology in evaluating spermatogenesis exceeds $90 \%$ in most studies (Table1). Turek et al correlated between FNA and biopsy in sperm detection for 34 sites and found that FNA showed sperm at all sites where biopsy predicted sperm presence but at three sites sperm was present on FNAC that was not predicted by biopsy and thus FNAC was found to be more sensitive than biopsy to detect presence of sperm in testis. ${ }^{23}$ Meng et al found discordant diagnosis between cytology and histology in $6 \%$ specimen. In half of these, the discordance was due to additional information provided by FNAC. ${ }^{16}$ It is likely that the thin sections performed during the histologic preparation "cut" tails of some spermatozoa, but these are well preserved when the whole FNA specimen is smeared on the glass. ${ }^{27}$

\section{FINE NEEDLE ASPIRATION MAPPING}

FNA mapping is defined as aspiration of more than four FNA sites per testis. ${ }^{23}$ This is performed most commonly as diagnostic test to detect presence and distribution of sperms in testis of men who have testicular atrophy or prior biopsy reveals abnormal or absent spermatogenesis. The idea behind FNA mapping is that there is geographic variation in presence of sperm in testes and these patches can be discovered by mapping even if biopsy shows absent sperm. Data from 59 
Jha et al. Testicular Fine Needle Aspiration in Evaluation of Male Infertility

nonobstructive azoospermic patients who underwent mapping and concurrent or prior testis biopsy revealed $27.1 \%$ likelihood that the fine needle aspiration map would show sperm despite a biopsy negative for sperm. ${ }^{28}$ FNA mapping is done under local anesthesia. The planned aspiration sites are marked over the scrotal skin. 2-11 aspiration sites, $5 \mathrm{~mm}$ apart, are marked on each testes depending upon the testicular size. FNA is performed with sharp beveled, 1 inch $23 \mathrm{G}$ needle with $10 \mathrm{ml}-20 \mathrm{ml}$ syringe attached to it. Precise gentle in and out movement, varying from $5-8 \mathrm{~mm}$ are used. 10-30 needle excursions are made at each site and slides are prepared from the aspirated material. Pressure is applied at each site for haemostasis. Aseptic technique should be applied during the procedure. ${ }^{25}$ Patient with suspected obstruction should not be mapped theoretically to avoid risk of multiple needle injury to normal or potentially reconstructable reproductive tract.

Table 1. Correlation between testicular fine needle aspiration and biopsy obtained in different studies in evaluating spermatogenesis

\begin{tabular}{|c|c|c|}
\hline STUDY( Year) & $\begin{array}{l}\text { NUMBER } \\
\text { OF } \\
\text { PATIENTS }\end{array}$ & $\begin{array}{c}\text { CYTOLOGIC } \\
\text { HISTOLOGIC } \\
\% \\
\text { AGREEMENT }\end{array}$ \\
\hline $\begin{array}{l}\text { Gottschak- Sabbag S } \\
(1993)^{25}\end{array}$ & 47 & $87 \%$ \\
\hline Mallidis $C(1994)^{12}$ & 46 & $94 \%$ \\
\hline Craft I $(1997)^{13}$ & 19 & $84 \%$ \\
\hline Odabas O $(1997)^{14}$ & 24 & $90 \%$ \\
\hline Mahajan AD(1999) ${ }^{15}$ & 60 & $97 \%$ \\
\hline Rammou- Kinia R(1999) ${ }^{6}$ & 30 & $87 \%$ \\
\hline Meng MV(2001) ${ }^{16}$ & 87 & $94 \%$ \\
\hline Qublan HS (2002) ${ }^{3}$ & 34 & $96 \%$ \\
\hline Aridogan IA $(2003)^{17}$ & 40 & $90 \%$ \\
\hline Srivastava A $(2004)^{18}$ & 46 & $95.6 \%$ \\
\hline $\operatorname{Han} U(2006)^{2}$ & 29 & $94 \%$ \\
\hline
\end{tabular}

\section{TESTICULAR ASPIRATION IN ASSISTED REPRODUCTION}

Before introduction of intracytoplasmic sperm injection (ICSI) in 1992, in which a single sperm was injected into the cytoplasm of a retrieved oocyte, the only available option for men with azoospermia was adoption or sperm donor. ${ }^{5}$ While in obstructive cases spermatozoa are abundant in the testes or epididymis, permitting a high retrieval rate, in NOA the epididymis is devoid of spermatozoa and only few foci of spermatogenesis are found in the testis. The concept of using testicular sperm to achieve fertilization and pregnancy has revolutionized the potential to treat patients with NOA. ${ }^{29,30}$ Testicular sperm extraction (TESE) procedure requires to remove a single large piece of testicular tissue to up to 20 small pieces of tissue which is then processed to obtain sperm for use in In vitro fertilization (IVF). ${ }^{31,32}$ Still chance of locating sperm in these testes by TESE is at best 40$60 \% .^{33-36}$ Complicating this problem is that recognized clinical parameters, including testicular size, history of ejaculated sperm, serum hormone levels or testis biopsy histology do not predict accurately whether sperm will be recovered during testicular biopsy for use in IVF or not. Also spermatogenesis may vary geographically within the failing testis, so precisely locating area of spermatogenesis is difficult. ${ }^{37-39}$ Failure to retrieve sperm from the testis in azoospermic subjects may cause him and his partner financial and psychological distress due to cancellation of IVF cycle, especially when sperm recovery techniques are performed simultaneously with ovarian stimulation of the female partner. An accurate preoperative assessment is thus beneficial both to the couple and reproductive operators. Testicular fine needle aspiration is useful in assisted reproduction in two ways- first, FNA mapping can locate the area of spermatogenesis in failing testis and thus biopsy for sperm retrieval can be directed to that particular site, second, fine needle aspiration itself can be used for sperm retrieval instead of biopsy. As sperm production within the testis can now be "mapped" with a fine needle, incision free technique and because of the large emotional and financial costs associated with IVF and ICSI, it has become a diagnostic challenge to fully inform the couple of the likelihood that sperm will be obtained from testes. The diagnostic FNA map may be vary informative to precisely locate mature sperm within the testis. This knowledge converts a random procedure into a more refined and directed technique that may minimize the amount of valuable tissue removed from failing testes. Prior reports of TESE procedures requiring 20 biopsies per patient and complete organ devascularization certainly lend weight to the goal of a "testis-sparing" approach. ${ }^{32,40}$ If FNA map do not reveal sperm then couple may be informed that TESE may or may not be able to retrieve sperm for ICSI and thus they may be mentally prepared to use donor sperm as backup and this may prevent IVF cycle cancellation. ${ }^{22}$

Retrieval of spermatozoa in case of obstructive azoospermia with use of percutaneous epididymal fine needle aspiration was reported by Shrivastava et al in 1994. ${ }^{41}$ First clinical application of testicular fine needle aspiration (TEFNA) for recovery of mature spermatozoa in NOA was reported by Lewin et al in 1996 and a pregnancy resulting in birth was achieved. In their study spermatozoa were retrieved by 21-23 G butterfly needle. They emphasized that FNA enable operator to reach more testicular area than open biopsy and thus increase the chance of retreiving spermatozoa for 
Jha et al. Testicular Fine Needle Aspiration in Evaluation of Male Infertility

ICSI. ${ }^{42}$ However other studies show that more number of sperms are recovered by TESE then by fine needle aspiration. ${ }^{19,20,43,44}$ Review done on different methods of sperm retrieval showed that TESE with multiple biopsies had higher sperm retrieval rate than FNA particularly in SCO syndrome and maturation arrest. ${ }^{45}$ But no significant difference in either fertilization rate or implantation and ongoing pregnancy rate was noted by spermatozoa received by either open biopsy or fine needle aspiration. ${ }^{21}$

\section{COMIPLICATIONS OF TESTICULAR FINE NEEDLE ASPIRATION COMIPARED WITH TESE}

Although the ultimate choice of testicular sperm retrieval methods will depend on a number of factors including the clinical diagnosis, patients' preference and the availability of the necessary surgical skills, one of the most important parameters will be the physiological consequences of these techniques on testicular function. Schlegel and Su evaluated 64 patients after TESE. ${ }^{40}$ At three months after TESE, $82 \%$ of evaluated patients had ultrasonographic abnormalities in the testis suggesting resolving inflammation or haematoma at the biopsy site. By six months, these acute inflammatory changes typically resolved leaving linear scars or calcifications. Two patients had documented impaired testicular blood flow, with complete devascularization of the testis for one patient after TESE with multiple biopsies. Another study demonstrated sonographic evidence of intratesticular bleeding in four out of $58(7 \%)$ percutaneous biopsies performed with 18 gauge biopsy needle, resolving within six months post-operatively..$^{46}$ On the other hand, no major complications were recorded, beside mild pain and discomfort by use of fine needle aspiration in most studies though one case of testicular bleeding following fine needle aspiration was found in another study. ${ }^{28,43,47,48}$ Westerland et al studied the consequences after testicular sperm aspiration (TESA) using suction and 19 gauge needle. Focal testicular lesions were seen in four out of 61 testes $(6.6 \%)$ at the three month investigation point. Three lesions resolved after six months and all after nine months. Testicular echogenicity remained unchanged in 50 cases $(82 \%)$ three months after TESA. Four men $(11.4 \%)$ reported severe subjective discomfort post-operatively, but only one had a medical consultation where an intratesticular haematoma was diagnosed. There were no significant changes in $\mathrm{FSH}$ and testosterone after procedure and testicular volumes were similar after three months. ${ }^{49}$ Shufaro et al studied impact of repeated TEFNA and TESE on the microscopic morphology of the testis in animal model. They found that TESE caused chronic inflammation, occasional necrosis and degenerative changes in testicular germ cells in only approximately ten percent of the remaining testicular tissue where as TEFNA caused widespread architectural distortion of seminiferous tubules into irregular and deformed lumens lined by Sertoli cells only, as well as focal chronic inflammation, necrosis and degenerative changes accompanied by decreased spermatogenesis. Similar but less extensive changes were noted when fewer punctures were performed. When negative suction pressure was not applied during TEFNA, similar histological changes occurred, indicating that testicular damage was related to the puncture itself. Following either procedure, the contralateral non-operated testes were unaffected. However the authors accept that the clinical relevance of such findings in testes of azoospermic men is yet to be determined. ${ }^{50}$

\section{ADVANTAGES OF FNAC}

FNAC is less invasive and gives informative data on spermatogenesis of entire testes. Report can be issued quickly as compared to biopsy. Complications related to procedure are rare. It is simple, quick and inexpensive because surgical instruments are not required. ${ }^{51}$ Local scarring doesn't occur. ${ }^{15}$ It is well tolerated by patient. Infertile patients feel more secure with aspiration than with biopsy. ${ }^{2}$ The material shows excellent preservation and various cell types can be identified. ${ }^{7}$ FNAC guided TESE is useful alternative to blind biopsy. ${ }^{22}$ Good concordance has been observed between histology and cytology (Table1). Material obtained can be used for quantitation of spermatogenesis by DNA Flow cytometry. ${ }^{52}$ -

\section{LIMITATIONS OF FNAC}

FNAC is unable to provide architectural information of testes. It doesn't give information about thickness of tubular basement membrane, tubular diameter or status of interstitial tissue. ${ }^{53}$ Testicular disorders leading to azoospermia such as atrophy, fibrosis and Leydig cell hyperplasia can be diagnosed on basis of histology but are difficult to assess by FNA. ${ }^{3}$ Some patients complain of prolonged pain but this can be relieved by scrotal support and analgesics. ${ }^{2}$ Fairly experienced pathologist is needed to interpret the smears. ${ }^{6}$ Neurogenic shock have been reported in patients who failed to rest after the procedure. Hematoma formation can be expected when thick needle (20G) is used. ${ }^{7}$

\section{CONCLUSIONS}

In spite of limitations, testicular FNAC represent an important diagnostic procedure in azoospermic men and a reliable prognostic parameter for successful sperm retrieval at TESE, particularly in NOA. Also sperm may be retrieved with fine needle aspiration alone for use in assisted reproduction. As assisted reproduction is gaining popularity in Nepal also, pathologists and urologists here also should develop skill and experience in performing the procedure and interpreting the reports in male infertility. 
Jha et al. Testicular Fine Needle Aspiration in Evaluation of Male Infertility

\section{REFRERENCES}

1. Sigman M, Jarow JP. Male infertility. In: Walsh PC, Retik AB, Vaughan ED, Weij AJ, Kavoussi LR, Norvick AC, et al, editors. Campbell's urology. 8th ed. Philadelphia, WB Saunders, 2003: p.1476.

2. Han U, Adabag A, Koybasioglu F, Onal BU. Clinical value of cell count and indices in Testicular Fine needle aspiration cytology in Primary Infertility - Diagnostic Performances compared with histology. Anal Quant Cytol Histol 2006;28(6):331-36.

3. Qublan HS, Al Jader KM, Al Kaisi NS, Alghoweri AS, Abu Khait SA, Abu Qamar AA, Haddadin E. Fine needle aspiration cytology compared with open biopsy histology for diagnosis of azoospermia. J Obstet Gynaecol 2002;22(5):527-31.

4. Jarow JP, Espeland MA, Lipshultz LI. Evaluation of the azoospermic patients. J Urol 1989;142:62-5.

5. Palermo G, Joris H, Devroey P, Van Steirteghem AC. Pregnancies after intracytoplasmic injection of single spermatozoon into an oocyte. Lancet 1992;340:17-8.

6. Rammou-Kinia R, Anagnostopoulou I, Tassiopoulos F, Lykourinas M. Fine needle aspiration of the testis. Correlation between cytology and histology. Acta Cytol 1999;43:991-8.

7. Al Jitawi SA, Al ramahi SA, Hakooz BA. Diagnostic role of testicular fine needle aspiration in male infertility. Acta cytol 1997;4:1705-8

8. Balselv E, Francis D, Jacobsen GK. Testicular germ cell tumors, Classification based on fine needle aspiration biopsy. Acta cytol 1990;34:690-94.

9. Lopez JI, Aranda FI. Fine needle aspiration cytology of spermatocytic seminoma. Acta cytol 1989;33:627-30.

10. Schenk U, Schill WB. Cytology of human seminiferous epithelium. Acta cytol 1988;32:689-96.

11. Papic Z, Katona G, Skrabalo Z. The cytologic identification and quantification of testicular cell subtypes: Reproducibility and relation to histologic findings in the diagnosis of male infertility. Acta cytol 1988;32:697-706.

12. Mallidis C, Gordon Baker HW. Fine needle tissue aspiration biopsy of the testis. Fertil Steril 1994;61:367-75.

13. Craft I, Tsirigotis M, Courtauld E, Farrer-Brown G. Testicular needle aspiration as an alternative to biopsy for the assessment of spermatogenesis. Hum Reprod 1997; 12(7):1483-7.

14. Odabas O, Urgas S, Aydin S, Yilmaz Y. Assessment of testicular cytology by fine needle aspiration and the imprint technique: are they reliable diagnostic modalities? Br J Urol 1997;79 (3):445-8.

15. Mahajan AD, Ali NI, Walwalkar SJ, Rege JD, Pathak HR. The role of fine needle aspiration cytology of the testis in the diagnostic evaluation of infertility. BJU Int 1999; 84(4):485-8.

16. Meng MV, Cha I, Ljung BM, Turek PJ. Testicular Fine-Needle Aspiration in Infertile Men: Correlation of Cytologic Pattern With Biopsy Histology. Am J Surg Pathol 2001;25(1):71-79.

17. Aridogan IA, Bayazit Y, Yaman M, Ersoz C, Doran S. Comparison of fine-needle aspiration and open biopsy of testis in sperm retrieval and histopathologic diagnosis. Andrologia 2003;35(2):121-5.

18. Srivastava A, Raghavendran M, Jain M, Gupta S, Chaudhary H. Fine-Needle Aspiration Cytology of the Testis: Can It Be a Single Diagnostic Modality in Azoospermia? Urol Int 2004;73(1):23-27.

19. Hauser R, Yogev L, Paz G, Yavetz H, Azem F, Lessing JB, et al. Comparison of Efficacy of Two Techniques for Testicular Sperm Retrieval in Nonobstructive Azoospermia: Multifocal Testicular Sperm Extraction Versus Multifocal Testicular Sperm Aspiration. J Androl 2006;27(1):28-33.

20. Mercan R, Urman B, Alatas C, Aksoy S, Nuhoglu A, Isiklar $\mathrm{A}$, et al. Outcome of testicular sperm retrieval procedures in non-obstructive azoospermia: percutaneous aspiration versus open biopsy. Hum Reprod 2000;15(7):1548-51.

21. Tournaye H, Clasen K, Aytoz A, Nagy Z, Van Steirteghem A, Devroey P. Fine needle aspiration versus open biopsy for testicular sperm recovery: a controlled study in azoospermic patients with normal spermatogenesis. Hum Reprod 1998;13:901-4.

22. Turek PJ, Givens C, Schriock ED, Meng MV, Pederson RA, Conaghan J. Testis sperm extraction and intracytoplasmic sperm injection guided by prior fine needle aspiration mapping in nonobstructive azoospermia. Fertil Steril 1999;71:552-7.

23. Turek PJ, Cha I, Ljung BM. Systematic fine-needle aspiration of the testis: correlation to biopsy and results of organ "mapping" for mature sperm in azoospermic men. Urology 1997;49(5):743-8.

24. Meng MV, Cha I, Ljung BM, Turek PJ. Relationship between classic histologic pattern and sperm findings on fine needle aspiration map in infertile men. Hum Reprod 2000;15(9):19737.

25. Gottschalk-Sabag S, Glick T, Weiss DB. Fine needle aspiration of the testis and correlation with testicular open biopsy. Acta Cytol 1993;37:67-72.

26. Batra VV, Khadgawat R, Agarwal A, Krishnani N, Mishra SK, Mithal A, et al. Correlation of cell counts and indices in testicular FNAC with histology in male infertility. Acta Cytol 1999;43:617-23.

27. Carpi A, Menchini Fabris FG, Palego P, Di Coscio G, Romani R, Nardini V, et al. Fine-needle and large-needle percutaneous aspiration biopsy of testicles in men with nonobstructive azoospermia: safety and diagnostic performance. Fertil Steril 2005;83(4):1029-33.

28. Turek PJ, Ljung BM, Cha I, Conaghan J. Diagnostic findings from testis fine needle aspiration mapping in obstructed and non-obstructed azoospermic men. J Urol 2000; 163:1709-16.

29. Schoysman R, Vanderzwalmen P, Nijs M, Segal L, Segal Bertin G, Geerts L, et al. Pregnancy after fertilization with human testicular spermatozoa (Letter). Lancet 1993;342:1237.

30. Craft I, Bennett V, Nicholson N. Fertilising ability of testicular spermatozoa. Lancet 1993; 342:864.

31. Schelgel PN, Palermo GD, Goldstein M, Menendez S, Zaninovic N, Veeck LL, et al. Testicular sperm extraction 
Jha et al. Testicular Fine Needle Aspiration in Evaluation of Male Infertility

with intracytoplasmic sperm injection for nonobstructive azoospermia. Urology 1997:49:435-40.

32. Tournaye H, Lui J, Nagy PZ. Correlation between testicular histology and outcome after intracytoplasmic sperm injection using testicular spermatozoa. Hum Reprod 1996;11:127-32.

33. Su LM, Palermo GD, Goldstein M, Veeck LL, Rosenwaks Z, SchlegelPN. Testicular spermextraction with intracytoplasmic sperm injection for nonobstructive azoospermia: testicular histology can predict success of sperm retrieval. J Urol 1999;161:112-6.

34. Seo JT, Ko WJ. Predictive factors of successful testicular sperm recovery in non-obstructive azoospermia patients. Int J Androl 2001;24:306-10.

35. Amer M, Ateyah A, Zohdy W, Abd El Nasser T, Abdel-Malak G, Fakhry E. Preoperative and intraoperative factors that predict difficult testicular sperm retrieval in patients with nonobstructive azoospermia. Fertil Steril 2002; 78:646-7.

36. Glander HJ, Horner LC, Dorschner W, Paasch U, Kratzsch J. Probability to retrieve testicular spermatozoa in azoospermic patients. Asian J Androl 2000; 2:199-205.

37. Bettella A, Ferlin A, Menegazzo M, Ferigo M, Tavolini $\mathrm{IM}$, Bassi PF, et al. Testicular fine needle aspiration as a diagnostic tool in non-obstructive azoospermia. Asian J Androl 2005;7:289-94.

38. Ezeh UI, Moore HD, Cooke ID. Correlation of testicular sperm extraction with morphological, biophysical and endocrine profiles in men with azoospermia due to primary gonadal failure. Hum Reprod 1998;13:3066-74.

39. Tournaye H, Verheyen G, Nagy P, Ubaldi F, Goossens A, Silber S, et al. Are there any predictive factors for successful testicular sperm recovery in azoospermic patients? Hum Reprod 1997;12:80-6.

40. Schlegel P, Su L. Physiological consequences of testicular sperm extraction. Hum Reprod 1997;12:1688-92.

41. Shrivastav P, Nadkarni P, Wensvoort S, Craft I. Percutaneous epididymal sperm aspiration for obstructive azoospermia. Hum Reprod 1994;9:2058-61.

42. Lewin A, Weiss DB, Friedler S, Ben-Sachar I, Porat -Katz A, Meirow D, et al. Delivery following intracytoplasmic injection of mature sperm cells recovered by testicular fine needle aspiration in a case of hypergonadotropic azoospermia due to maturation arrest. Hum Reprod 1996;11:769-71.
43. Friedler S, Raziel A, Strassburger D, Soffer Y, Komarovsky D, Ron-El R. Testicular sperm retrieval by percutaneous fine needle sperm aspiration compared with testicular sperm extraction by open biopsy in men with non-obstructive azoospermia. Hum Reprod 1997;12:1488-93.

44. Ezeh O, Moore H, Cooke I. A prospective study of multiple needle biopsies versus a single open biopsy for testicular sperm extraction in men with non obstructive azoospermia. Hum. Reprod 1998;13:3075-80.

45. Donoso P, Tournaye H, Devroey P. Which is the best sperm retrieval technique for non-obstructive azoospermia? A systematic review. Hum Reprod Update,2007;13(6):539-49.

46. Lewin A, Reubinoff B, Porat-Katz A, Weiss D, Eisenberg V, Arbel R, et al. Testicular fine needle aspiration: the alternative method for sperm retrieval in non-obstructive azoospermia. Hum Reprod 1999;14:1785-90.

47. Harrington TG, Schauer D, Gilbert BR. Percutaneous testis biopsy: an alternative to open testicular biopsy in the evaluation of the subfertile man. J Urol 1996;156:1647-51.

48. Watkins W, Nieto F, Bourne H. Testicular and epididymal sperm in a microinjection program: methods of retrieval and results. Fertil Steril 1997;67:527-35.

49. Westlander G, Erling E, Seth G, Nils L, Lars N, Charlotte W, et al. Serial ultrasonography, hormonal profile and antisperm antibody response after testicular sperm aspiration. Hum Reprod 2001;16(12):2621-7.

50. Shufaro Y, Prus D, Laufer N, Simon A. Impact of repeated testicular fine needle aspirations (TEFNA) and testicular sperm extraction (TESE) on the microscopic morphology of the testis: an animal model. Hum Reprod 2002;17(7):1795-9.

51. Hussein MR, Bedaiwy MA, Ezat A, Ibraheem AF, Nayel M. Role of Fine Needle aspirate Mapping and Touch Imprint Preparations in evaluation of azoospermia. Anal Quant Cytol Histol 2005;27(2):67-70.

52. Dey P, Mondal AK, Singh SK, Vohra H. Quantitation of spermatogenesis by DNA flow cytometry from fine-needle aspiration cytology material. Diagn Cytopathol 2000; 23(6):386-7.

53. Dusmez D, Aydin O, Akbay E, Cayan S, Kanik EA. Touch Imprint Cytology in Biopsy of Infertile Testis. Acta Cytol 2001;45 (6):990-4. 